\title{
The effect of cyclic rifaximin therapy on symptoms of diverticular disease from the perspective of the gastroenterology outpatient clinic: a "real-life" study
}

\author{
Andrzej Moniuszko ${ }^{1}$, Grażyna Rydzewska ${ }^{1,2}$ \\ ${ }^{1}$ Department of Gastroenterology with Inflammatory Bowel Disease Subdivision, Central Clinical Hospital of the Ministry \\ of Interior and Administration, Warsaw, Poland \\ ${ }^{2}$ Faculty of Medicine and Health Sciences, Jan Kochanowski University, Kielce, Poland
}

Gastroenterology Rev 2017; 12 (2): 145-151

DOI: https://doi.org/10.5114/pg.2017.68167

Key words: diverticular disease, rifaximin, cyclic therapy.

Address for correspondence: Andrzej Moniuszko MD, PhD, Department of Gastroenterology with Inflammatory Bowel Disease Subdivision, Central Clinical Hospital of the Ministry of Interior and Administration, 137 Woloska St, Warsaw, Poland, e-mail: moniuszko.a@gmail.com

\begin{abstract}
Introduction: Symptomatic uncomplicated diverticular disease of the colon (SUDD) is one of the most common diseases with which patients present to a gastroenterologist. Mild forms of diverticulitis can also be treated using rifaximin. Although numerous randomised controlled trials have already demonstrated the efficacy of rifaximin therapy, there is still a lack of data from daily medical practice.

Aim: To assess the effect of rifaximin on the symptoms of diverticular disease (SUDD and mild diverticulitis) in patients undergoing routine treatment in gastroenterology outpatient clinics in Poland.

Material and methods: The retrospective study included 142 patients with a diagnosis of SUDD and mild diverticulitis, with a mean age of 60-69 years (41\%), of whom 65\% were women. Patients underwent three cycles of rifaximin therapy at a dose of $2 \times 400 \mathrm{mg}$ daily for 7 days over 3 consecutive months. Survey data were collected during monthly clinic appointments using a questionnaire completed by 48 gastroenterologists, and in selected cases standard inflammatory parameters were also determined.

Results: After just one cycle of therapy a significant reduction in disease symptoms was observed (abdominal pain, abdominal tenderness, bloating, disturbances in bowel habit), defined over a scale of 0-3 points. The mean intensity of symptoms decreased from $1.7 \pm 0.7$ to $0.8 \pm 0.3$ points (with a maximum symptom intensity of 3.0 points). After three cycles, the severity of symptoms decreased markedly to an average of $0.3 \pm 0.1$, and as many as $75 \%$ of patients reported no abdominal pain (previously the percentage was only $4 \%$ ). These differences were statistically significant, $p<0.001$. The decrease in inflammatory parameters (white blood cell count, C-reactive protein and erythrocyte sedimentation rate) was statistically significant.

Conclusions: Rifaximin is highly effective in the symptomatic relief of uncomplicated diverticular disease of the large bowel, and it is also effective in the treatment of mild forms of diverticulitis. Although the effects were already visible after the first cycle of therapy, the highest efficacy was obtained after three cycles of therapy. Rifaximin can be successfully used in routine medical practice.
\end{abstract}

\section{Introduction}

Diverticular disease is one of the main diseases that gastroenterologists and surgeons face in their day-today practice. In Western Europe and North America, the cost of treating diverticular disease and its complications ranks fifth in the global scale of gastroenterology costs. This disease affects people of all ages, although it most often occurs in the older population. According to epidemiological data, $5 \%$ of the population aged
40 years, $30 \%$ at the age of 60 years, and $50-65 \%$ of the population aged 80 years are affected [1]. In most the population, the presence of diverticuli itself does not cause any symptoms; however, $20 \%$ of cases go on to develop symptomatic, uncomplicated diverticular disease (SUDD). The most common complication of diverticular disease is acute uncomplicated diverticulitis. According to recent studies, only about $4 \%$ of patients with diverticulosis have this complication [2]. 
Symptoms of SUDD include episodic bouts of abdominal pain, bloating, or disturbances in bowel habit, including diarrhoea, constipation, or alternation of these symptoms. The intensity and frequency of episodes of symptoms are individually variable, from mild to severe, strongly limiting the patient's activity and reducing their quality of life [2-6]. Treatments for diverticular disease include: a high-fibre diet, probiotics, symptomatic drugs, and antibiotics including mainly rifaximin, known for its eubiotic effects.

Rifaximin is a semi-synthetic antibiotic from the rifamycin group, which is minimally absorbed from the gut. Its broad spectrum of antibacterial activity and the high concentration it achieves in the gastrointestinal tract with minimal absorption to the bloodstream have made it widely used in gastroenterology [7]. Rifaximin has a broad spectrum of bactericidal activity including Gram-positive and Gram-negative bacteria, both aerobic and anaerobic. Pharmacokinetic studies have shown that less than $0.4 \%$ of the dose is absorbed into the blood stream, with a comparable level in the urine, whilst in bile or breast milk the level is undetectable, and $97 \%$ of the drug is excreted unchanged in the faeces $[7,8]$.

In addition to its typical antimicrobial activity, this drug has additional mechanisms of action, unmatched by other antibiotic groups. These include: modulation of the microflora of the gastrointestinal tract by promoting the growth of Lactobacilli and Bifidobacteria strains (the so-called "eubiotic" effect) as well as modulation of bacterial metabolism, including inhibition of the hydrocarbon-derived pathways $[8,9]$. This drug is also capable of reducing the virulence of enteropathogenic Escherichia coli strains by inhibiting the expression of enterotoxins or adhesive factors [10]. Interestingly, rifaximin is distinguished by several anti-inflammatory activities mainly exerted by the pregnane $X$ receptor (PXR), expressed primarily in the gastrointestinal tract, the small intestine, and the colon. Due to the activity described above, rifaximin is called a eubiotic, not an antibiotic. Rifaximin has been used in the treatment of travellers' diarrhoea, hepatic encephalopathy, small intestine bacterial overgrowth, irritable bowel syndrome, and diverticular disease.

The efficacy of the long-term use of cyclic rifaximin in the symptomatic treatment of uncomplicated diverticular disease (12-24 months) has been demonstrated in several randomised clinical trials [11-14] and a large meta-analysis in 2011 [15]. According to the current guidelines of the Polish Society of Gastroenterology and the Society of Polish Surgeons [16] in SUDD rifaximin should be used cyclically at a dose of $400 \mathrm{mg}$ daily for 7 days every month for a total of 12 months. Rifaximin together with a high dose of soluble fibre is also rec- ommended for the prevention of recurrent diverticulitis. The Italian Society issued detailed guidelines for the use of rifaximin in various stages of diverticular disease in February 2017 [17].

\section{Aim}

The main aim of the study was to evaluate the effects of rifaximin on the most common symptoms of diverticular disease in patients treated routinely in gastroenterology outpatient clinics.

\section{Material and methods}

The retrospective study included 142 patients with symptomatic, uncomplicated diverticular disease (SUDD). All the data on which this analysis was based were compiled using a questionnaire completed by a total of 48 gastroenterologists in their routine outpatient practice at the time of onset of rifaximin therapy, systematically supplemented on subsequent visits. The form included, among others, the patient's past medical history, age, time of diagnosis, diagnostic method on which the diagnosis of diverticular disease was based, the most common symptoms reported by the patient during the visit, past treatment, and dietary habits. In the group of patients with a suspicion of diverticulitis, standard laboratory tests of inflammatory factors, including erythrocyte sedimentation rate (ESR), C-reactive protein (CRP), and white blood cell count (WBC), were also included.

Sixty-five percent of the population were women. The largest subgroup of patients were patients aged $60-69$ years (41\%). In total $74 \%$ of all patients exceeded 60 years of age. In this group of patients, the diagnosis of diverticular disease had been made in more than $65 \%$ of cases in the previous 2 years, while in $30 \%$ of patients diverticular disease was diagnosed at the time of inclusion in the study. The presence of colonic diverticula was most often found in endoscopic examination, colonoscopy (94\%), abdominal computed tomography (CT) (64\%), and abdominal ultrasonography (US) (45\%). At the time of initiating the examination, patients were primarily complaining of abdominal pain, abdominal tenderness, diarrhoea, constipation, or varying rhythm of bowel movements and bloating. Abdominal pain was reported in as many as $96 \%$ of all patients, mainly in the lower left iliac fossa (56\%) or lower abdomen (30\%), most often chronic (Figure 1).

Prior to inclusion in the study, patients used a wide range of drugs, mainly antispasmodics (46\%, mainly drotaverine and mebeverine), and fibre preparations (36\%), probiotics (27\%), trimebutine (19\%), mesalazine (14\%), and antibiotics 4\% (mainly metronidazole). Only $22 \%$ of patients used a high-fibre diet. 


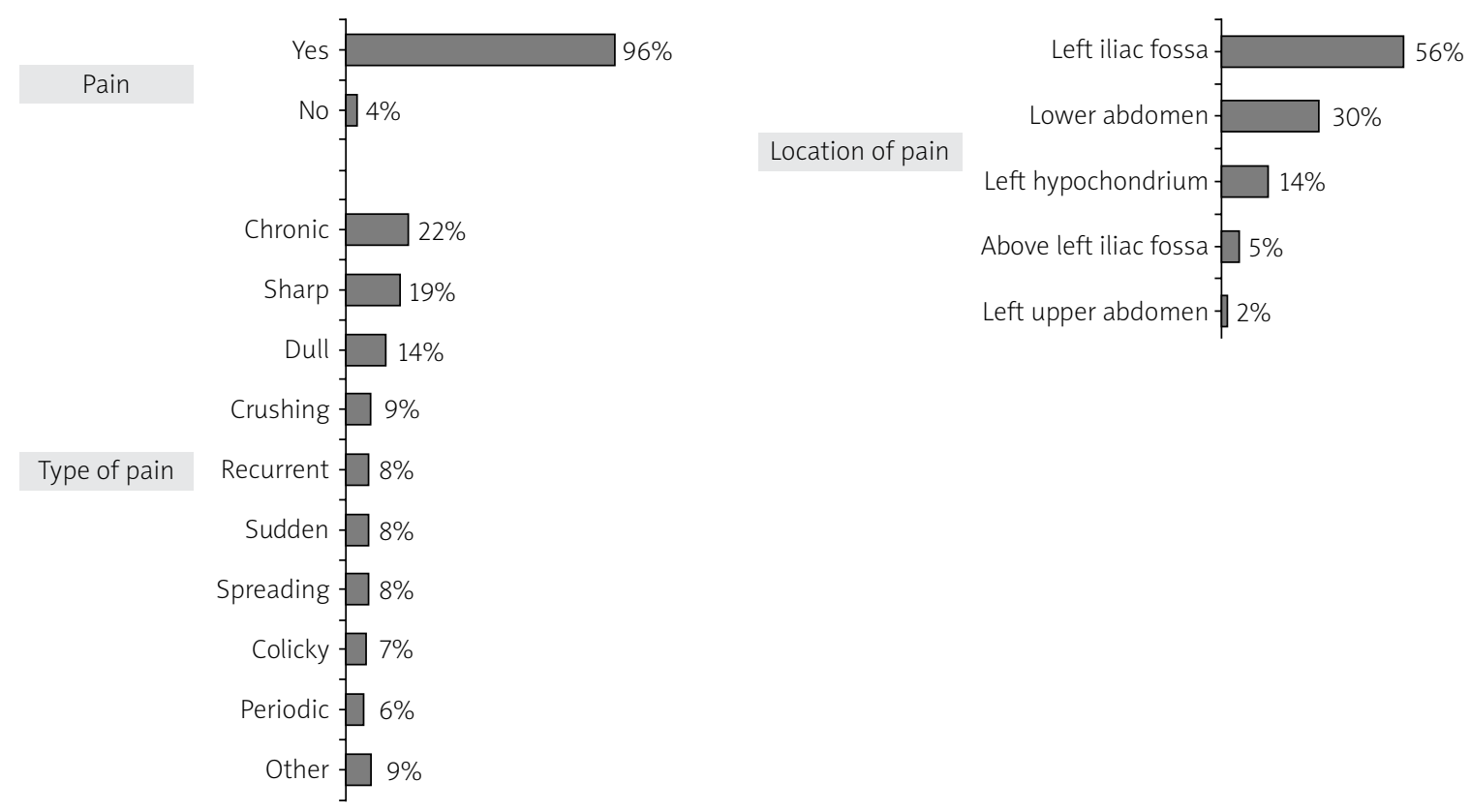

Figure 1. Characteristics and location of abdominal pain, visit 0

All patients received cyclic rifaximin therapy in accordance with the guidelines [16]. The patients were given oral rifaximin $800 \mathrm{mg} /$ day (two tablets b.d) for 7-10 days cyclically every month for 3 months. Based on interviews with the patient, the physician completed tables on the presence and severity of disease symptoms after each treatment cycle, including diet and supplementary medications, if taken. Gastrointestinal symptoms were assessed on a four-step scale, where 0 was a lack of symptoms, 1 indicated mild symptoms, 2 - moderate, and 3 was the highest symptom severity. In the final part of the form, doctors also answered the question of what measures could facilitate the diagnosis, treatment, and education of a patient with diverticular disease in their daily medical practice.

\section{Statistical analysis}

Statistical analysis included descriptive methods the variables were presented as a mean with standard deviation or medians with one and three quartiles in nonparametric distribution. The distribution of variables was determined using the Shapiro-Wilk test. Correlation of the parametric variables was calculated using the Student's $t$-test, and for nonparametric variables the Wilcoxon test. Levels of $p \leq 0.05$ were deemed to be statistically significant.

\section{Results}

After 3 months of rifaximin therapy, a significant reduction in symptoms was observed in the majority of patients, as shown in Figure 2. The mean severity of all symptoms (described using a four-step scale) reported by the patient on their visit prior to the inclusion of rifaximin was $1.7 \pm 0.7$ points (maximum 3 points).

After just one cycle of therapy a significant reduction of symptoms was observed - the mean intensity of all symptoms was $0.8 \pm 0.3$ points. After completion of three cycles of therapy, the mean severity of the symptoms further reduced to $0.3 \pm 0.1$ points (where the maximum symptom intensity was 3.0). The differences in intensity between visits were statistically significant $(p<0.001)$

A detailed analysis of the data is presented in Tables I and II. The most common and most bothersome symptom was abdominal pain. During their visit, $84 \%$ of patients reported significant pain intensity at a level of 2 and 3 points, on average 2.2, and only $4 \%$ of patients did not experience pain. Significantly, after three cycles of rifaximin therapy, this proportion was completely reversed - symptom-free patients accounted for as many as $75 \%$ of the total, and only $2 \%$ of the population reported pain of $\geq 2$ points.

An equally important symptom reported by patients was bloating - experienced at visit 0 by $99 \%$ of patients, of which $87 \%$ reported a severity of $\geq 2$ points (mean 2.3/3.0 pts). After three treatment cycles, almost two thirds (65\%) of the patients were completely asymptomatic, and 35\% described the severity of bloating at a level of 1 point (in total mean 0.4/3.0 points); the differences were statistically significant, $p<0.001$.

Substantial changes were also been observed in the context of diarrhoea. At the visit preceding the inclusion 


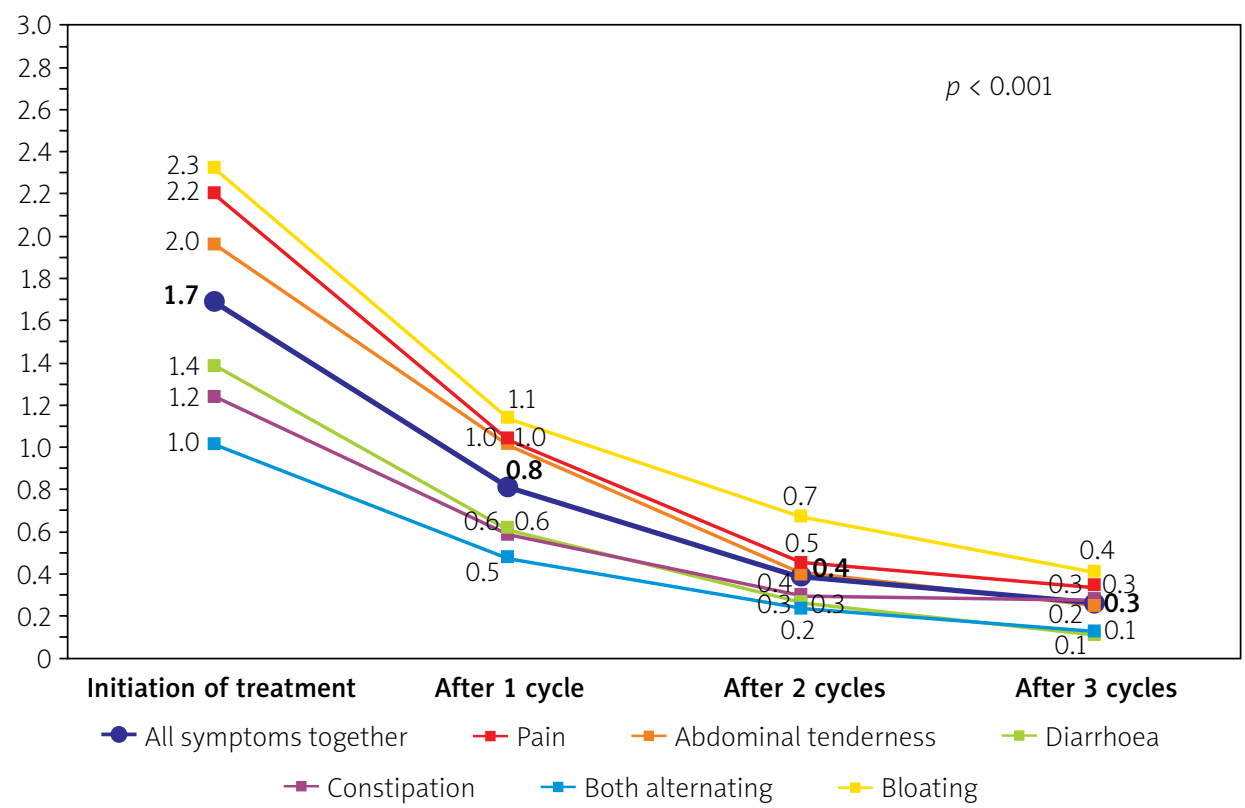

Figure 2. Changes in intensity of symptoms after $0,1,2,3$ cycles of therapy, sum of all symptoms shown in bold type

Table I. Characteristics and distribution of disease symptoms in population, visit 0

\begin{tabular}{lcccccc}
$\begin{array}{l}\text { Initiation of } \\
\text { treatment }\end{array}$ & Pain & $\begin{array}{c}\text { Abdominal } \\
\text { tenderness }\end{array}$ & Diarrhoea & Constipation & Both alternately \\
\hline Mean & 2.2 & 2.0 & 1.4 & 1.2 & 1.0 \\
\hline \begin{tabular}{l} 
Percentage of scale: \\
\hline 0
\end{tabular} & 4 & 4 & 33 & 39 & 51 & 1 \\
\hline 1 & 9 & 23 & 19 & 14 & 2.3 \\
\hline 2 & 52 & 47 & 25 & 31 & 27 & 40 \\
\hline 3 & 36 & 26 & 24 & 16 & 13
\end{tabular}

Intensity of symptoms: 0 - no symptoms, 1 - mild, 2 - moderate, 3 - severe.

Table II. Characteristics and distribution of disease symptoms in population, after 3 months of therapy

\begin{tabular}{|c|c|c|c|c|c|c|}
\hline After 3 months & Pain & $\begin{array}{l}\text { Abdominal } \\
\text { tenderness }\end{array}$ & Diarrhoea & Constipation & Both alternately & Bloating \\
\hline Mean & 0.3 & 0.2 & 0.1 & 0.3 & 0.1 & 0.4 \\
\hline \multicolumn{7}{|c|}{ Percentage of scale: } \\
\hline 0 & 75 & 78 & 90 & 75 & 88 & 62 \\
\hline 1 & 22 & 20 & 8 & 21 & 11 & 35 \\
\hline 2 & 1 & 2 & 2 & 4 & 1 & 3 \\
\hline 3 & 1 & 0 & 0 & 0 & 0 & 0 \\
\hline
\end{tabular}

Intensity of symptoms: 0 - no symptoms, 1 - mild, 2 - moderate, 3 - severe. 
of therapy, half of the patients (49\%) reported moderate or severe diarrhoea in the previous days defined as $\geq 2$ points (mean $1.4 / 3.0$ points), while one in three patients remained asymptomatic. After the completion of three cycles of therapy again the proportions were completely reversed: in $90 \%$ of patients the episodes of diarrhoea had subsided, the final intensity of the symptom was on average $0.1 / 3$ points, no patient reported severe diarrhoea defined as 3 points; the differences were statistically significant $(p=0.003)$.

Similarly, in the case of constipation: on the first visit preceding the inclusion of rifaximin, this affected $61 \%$ of the total of patients, $47 \%$ reporting moderate and severe $\geq 2 / 3$ points symptoms, the mean intensity was $1.2 / 3$. After three cycles of therapy only $25 \%$ of patients still complained of constipation, but only $4 \%$ of them reported the symptom intensity as being moderate or severe, the mean symptom intensity was $0.3 / 3$ points, and the differences had a statistical significance of $p<0.001$.

Figure 3 shows the results of inflammatory parameters in the selected group of patients. At the time of inclusion in the study, 54\% had elevated WBC > 10,000. White blood cell count (median: 11,000 (8.23-13.0)), elevated ESR in 55\% (median: $30.0 \mathrm{~mm}$ (16.5-46.5)), and serum C-reactive protein concentration in the peripheral blood raised in 89\% (median: $13.2 \mathrm{mg} / \mathrm{dl}$ (6.65-27.5)). After 3 months of therapy these parameters were significantly normalised: respectively a normal WBC count was found in 97\% patients (median: 7100 (5.8-8.0)), normal ESR - in 89\% (median: $13 \mathrm{~mm}$ (10.0-20.0)), and CRP - in 36\% (median: $4.5 \mathrm{mg} / \mathrm{dl}$ (3.0-6.0)). The above differences were statistically significant $(p<0.001)$.
During the study, a reduction in drug intake was also observed: the need for antispasmodics decreased from $46 \%$ to $26 \%$, trimebutine - from $19 \%$ to $12 \%$. Mesalazine was only used by $4 \%$ of the previous $14 \%$ of patients, antibiotics other than rifaximin: 0 out of $4 \%$, probiotics: $18 \%$ out of $27 \%$; the use of soluble fibre preparations did not change.

\section{Discussion}

The results obtained in this study strongly confirm the efficacy of rifaximin in reducing the symptoms of uncomplicated diverticular disease as well as in the treatment of uncomplicated diverticulitis in the Polish population. Similar results were demonstrated by Colecchia et al. in a study carried out in 2007 [11], in which a much longer, 2-year period of cyclic rifaximin therapy was employed. In this study, $87.5 \%$ of patients reported abdominal pain at the initial visit; after 12 months of cyclic therapy only $17.2 \%$ and after 24 months $-12.9 \%$. A similar observation was reported in the case of bloating.

The results obtained in our study are highly consistent with the results of the largest analysis so far conducted by Stallinger et al. [18]. This was also an observational study conducted in a private health care system in Austria. The study included over 1000 patients with uncomplicated symptomatic diverticular disease (SUDD), who had experienced a minimum of three episodes of aggravation of symptoms over the previous year. The follow-up period was also 3 months, so the efficacy of the treatment was evaluated after three full cycles of rifaximin therapy. There was a statistically significant remission of all the symptoms typ-
Initiation of treatment

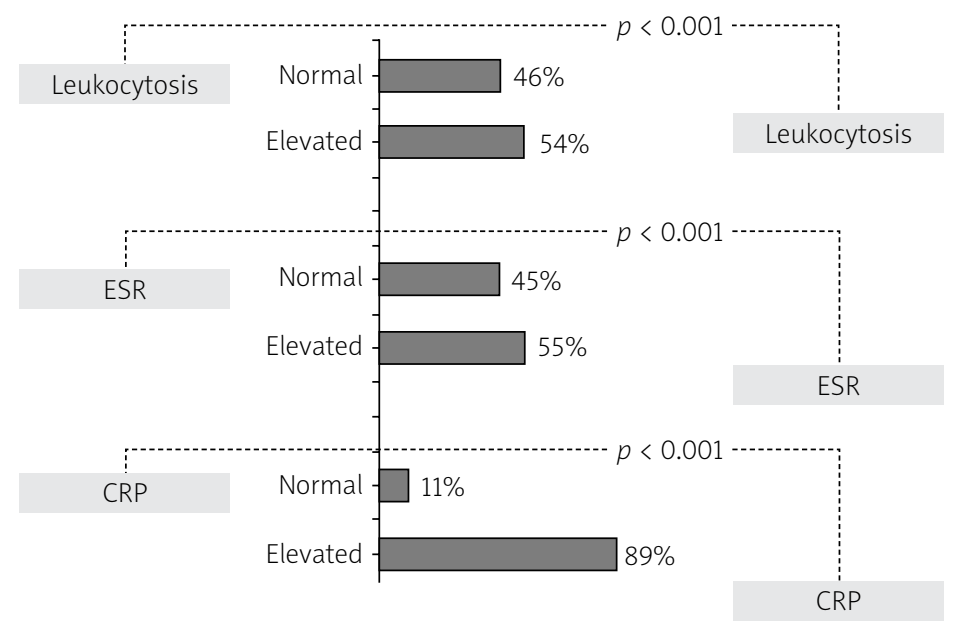

After 3 months of therapy

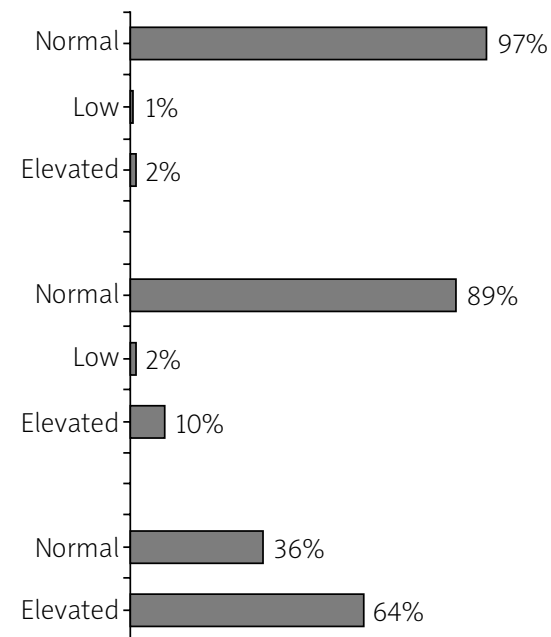

Figure 3. Changes in concentration of standard inflammatory parameters (WBC, ESR, CRP), visit 0 and after 3 months of therapy 
ical for diverticular disease that had been reported by the patients - including pain, bloating, and bowel habit disturbance. There was also a high rate of compliance, i.e. the percentage of patients taking the medication regularly, as recommended by the doctor.

Interestingly, in the Stallinger study more than $2 / 3$ of the study population had not received any prior medication. In turn, in our observation, most patients prior to inclusion in the study had received several symptomatic preparations, ranging from antispasmodics, through herbal remedies and mesalazine, to antibiotics, with moderate effect. Moreover, in more than $60 \%$ of Polish patients, diverticular disease had been diagnosed more than 2 years previously. It can be concluded from this that the patients included in the observation had a more severe course of the disease, not responding to standard treatment. We have shown that the use of rifaximin in this population was highly effective and led to a significant reduction in the intake of all other drug classes.

The results of both studies allow a full evaluation of the efficacy of this drug; moreover, conducting our study in an outpatient setting may give important practical information for gastroenterologists working in Poland. On the one hand, the methods used in our study cannot be compared with randomised clinical trials, in which the study drug is evaluated concurrently versus placebo or another drug. However, generally the criteria for inclusion in a clinical trial greatly limit the population of patients, whereas in daily clinical practice the treatment group often looks very different. Hence, documenting the beneficial effect of rifaximin in a "real life" study may also be of importance in everyday clinical practice. In addition, every day in medical practice we meet patients who, despite recommendations, still use many other preparations, especially over-the-counter remedies, including probiotics and symptomatic drugs. The results of our work indirectly demonstrate the efficacy of rifaximin also in the context of a significant reduction in the intake of these preparations.

The safety profile of rifaximin has already been extensively discussed in the registration studies of the preparation. In our study, the evaluation of complications was incomplete, and therefore it has been omitted in this analysis. In turn, in the above-mentioned, largest "real-life" outpatient study [18], the incidence of complications was very low, 24 adverse events in 20 of 1003 patients were recorded, of which only six adverse events showed a causal relationship to the use of rifaximin (0.6\% adverse drug reaction).

The efficacy of long-term rifaximin therapy in the treatment of symptomatic uncomplicated diverticular disease (12-24 months) has been demonstrated in sev- eral randomised clinical trials [11-15], although there is a lack of studies including a longer period of observation. Although it has been demonstrated that the use of rifaximin in SUDD reduces the risk of diverticulitis, as outlined in the current guidelines [16, 17], there are still no cost-effectiveness studies for this prophylaxis.

At the end of the study, gastroenterologists were also asked to answer several practical questions that were not covered in the Results section. One of the questions we asked was what would be useful in everyday medical practice to facilitate the diagnosis, therapy, and education of the patient. More than $30 \%$ of the physicians considered the cost of rifaximin and the lack of reimbursement of this preparation as a significant factor. Currently, unfortunately, not every patient can afford to finance cyclic therapy despite its efficacy. Reimbursement of rifaximin would help increase the access of patients with symptomatic uncomplicated diverticular disease to such effective treatment. A further $19 \%$ of respondents indicated that the low availability of imaging studies may be an obstacle to diagnosis, and $13 \%$ deem the availability of colonoscopy to be insufficient. According to almost half of the physicians, a greater amount of education materials regarding the disease itself and recommendations would be useful for patients, including dietary advice (47\%).

\section{Conclusions}

The results of this study confirm that rifaximin is effective in treating uncomplicated, symptomatic diverticular (SUDD) disease and uncomplicated diverticulitis in outpatient practice. Conducting a study under gastrointestinal clinic conditions allows us to conclude that by rapidly reducing symptoms such as abdominal pain, bloating, or disturbances in bowel habit, this drug can, from the first cycle of therapy, be successfully used in daily medical practice in a regimen of at least three cycles, ensuring a highly effective reduction of symptoms.

\section{Acknowledgments}

The study was sponsored by Alfa Wassermann.

\section{References}

1. Stępień B, Piotrowicz G, Rydzewska G. Leczenie choroby uchyłkowej jelita grubego z uwzględnieniem roli mesalazyny. Prz Gastroenterol 2013; 8: 211-7.

2. Bartoszek A, Domżał-Drzewicka R, Kachaniuk K, et al. The state of nutrition and the self-assessment of symptoms of depression in the group of seniors living in the countryside of Lublin province - preliminary report. Prz Gastroenterol 2015; 10: 208-14.

3. Shahedi K, Fuller G, Bolus R, et al. Long-term risk of acute diverticulitis among patients with incidental diverticulosis 
found during colonoscopy. Clin Gastroenterol Hepatol 2013; 12: 1609-13.

4. Strate LL, Modi R, Cohen E, et al. Diverticular disease as a chronic illness evolving epidemiologic and clinical insights. Am J Gastroenterol 2012; 107: 1486-93.

5. Bolster LT, Papagrigoriadis S. Diverticular disease has an impact on quality of life-results of a preliminary study. Colorectal Dis 2003; 5: 320-3.

6. Comparato G, Fanigliulo L, Aragona G, et al. Quality of life in uncomplicated symptomatic diverticular disease: is it another good reason for treatment? Dig Dis 2007; 25: 252-9.

7. Descombe JJ, Dubourg D, Picard M, Palazzini E. Pharmacokinetic study of rifaximin after oral administration in healthy volunteers. Int J Clin Pharmacol Res 1994; 14: 51-6.

8. Herbert L, DuPont MD. Biologic properties and clinical uses of rifaximin. Exp Opin Pharmacother 2011; 12: 293-302.

9. Hartmann G, Honikel KO, Knusel F, et al. The specific inhibition of the DNA-directed RNA synthesis by rifamycin. Biochim Biophys Acta 1967; 145: 843-4.

10. Jiang ZD, Ke S, DuPont HL. Rifaximin-induced alteration of virulence of diarrhoea-producing Escherichia coli and Shigella sonnei. Int J Antimicrob Agents 2010; 35: 278-81.

11. Colecchia A, Vestito A, Pasqui F, et al. Efficacy of long term cyclic administration of the poorly absorbed antibiotic rifaximin in symptomatic, uncomplicated colonic diverticular disease. World I Gastroenterol 2007; 13: 264-9.

12. Papi C, Ciaco A, Koch M, et al. Efficacy of rifaximin on symptoms of uncomplicated diverticular disease of the colon. A pilot multicentre open trial. Diverticular Disease Study Group. Ital J Gastroenterol 1992; 24: 452-6.

13. Papi C, Ciaco A, Koch M, et al. Efficacy of rifaximin in the treatment of symptomatic diverticular disease of the colon. A multi-centre double-blind placebo-controlled trial. Aliment Pharmacol Ther 1995; 9: 33-9.

14. Latella G, Pimpo MT, Sottili S, et al. Rifaximin improves symptoms of acquired uncomplicated diverticular disease of the colon. Int J Colorectal Dis 2003; 18: 55-62.

15. Bianchi M, Festa V, Moretti A, et al. Meta-analysis: long-term therapy with rifaximin in the management of uncomplicated diverticular disease. Aliment Pharmacol Ther 2011; 33: 902-10.

16. Pietrzak A, Bartnik W, Szczepkowski M, et al. Polish interdisciplinary consensus on diagnostics and treatment of colonic diverticulosis. Pol Przeg Chir 2015; 87: 203-20.

17. Cuomo R, Barbara G, Annibale B. Rifaximin and diverticular disease: position paper of the Italian Society of Gastroenterology (SIGE). Dig Liver Dis 2017 Feb 1. pii: S1590-8658(17)30192-5.

18. Stallinger S, Eller N, Högenauer C. Non-interventional study evaluating efficacy and tolerability of rifaximin for treatment of uncomplicated diverticular disease. Wiener Klin Wochenschr 2014; 126: 9-14.

Received: 17.05.2017

Accepted: 24.05.2017 